\title{
COVID-19 Mental Health Challenges: A Systematic Review, Logistic Regression and Principal Component Analysis
}

\author{
Savita J Gaur, Usha Panjwani* and Bhuvnesh Kumar \\ Department of Neurophysiology, Defence Institute of Physiology and Allied Sciences (DIPAS), India
}

Submission: May 27, 2020; Published: July 17, 2020

*Corresponding author: Usha Panjwani, Department of Neurophysiology, Defence Institute of Physiology and Allied Sciences (DIPAS), DRDO, Delhi -54 , India

\section{Abstract}

Background: World Health Organization (WHO)declaredCOVID-19 (SARS-COV2) viral infection as Pandemic on January 31, 2020. The virus is a highly transmittable and pathogenic, displaying primary symptoms of elevated temperature, cough, headache and to some extent loss of taste or smell. However, there are imperceptible psychological effects that are difficult to assess and cure than relevant physical indicators. COVID-19 Positive patients and Medical staff are at high risk to get affected psychologically. Therefore, the mental health status is required to be monitored on priority basis. This study aims to review, synthesize, and analyze published evidence on the prevalence of, anxiety, depression, and insomnia among patients, medical staff, and others (general population) during the COVID-19 outbreak.

Method: Based on PRISM Statement systematic search and review of published data till May 2020 on SARS-COV2 related psychological factor analysis was carried out. Logistic regression and PCA was performed to assess the prevalence of specific psychological aspects related to the prevailing COVID-19 pandemic.

Findings: The studies in included in the review, assessed anxiety, depression, distress, insomnia, and suicidal cases in relation to COVID-19. Participants reported psychological burden and high prevalence rates of depression, anxiety, and insomnia. The multiple linear regression analysis showed significance for anxiety $(\mathrm{p}<0.05)$. PCA reduced for critical Psych parameters into two components, PC1 with eigen value $(\varepsilon=2.67)$ and PC2 having eigen value $(\varepsilon=1.11)$.

Interpretation: COVID-19 patients, frontline medical staff, are at higher risk of mental health challenges.

Recommendation: The study recommends psychological prescription to COVID-19 patients, medical staff, and general population.

\section{Introduction}

COVID-19 or SARS-COV2is an infectious virus newly discovered in the coronavirus family. World Health Organization (WHO) declaredCOVID-19 (SARS-COV2) viral infection as Pandemic on January 31, 2020 [1]. The COVID-19 virus is mainly transmitted by coming in close proximity of a person who is COVID-19 positive. The virus spreads through droplets generated when a person coughs, sneezes, or exhales. To control the spread of viral infection around 4 billion i.e. half of the world population have been ordered by respective Governments to follow home confinement that is apparently the largest psychological experiment ever. Till date, Globally, there have been 4, 904, 413 confirmed cases of COVID-19, including 323,412 deaths, reported to WHO [1]. According to reported researched during epidemic and/or deadly illnesses there are enormous mental pressure on affected population. Social distancing, isolation, and fear of COVID-19, inadequate PPEs are making people vulnerable to mental health problems specifically on patients and frontline medical staff [2,3]. During quarantine, home confinement and while carrying out essential health care duties it's likely that one feels anxious, depressed and concurrently loses sleep [4]. Increased workload, physical exhaustion, inadequate and nosocomial transmission, fear of corona virus is supposed to elevate the most common emotion that is anxiety. Also, persons who had mental health history may face novel psychological challenges or their condition may worsen during Corona Quarantine. A dramatic decline in physical and mental well-being has also triggered some suicide cases related to COVID-19. Therefore, the Psychological support during COVID-19 Pandemic is vital towards enhancing resilience and good mental health. The aim this study is to carry out Review of COVID-19 related mental health issues among the affected population. We have compiled and evaluated data that gives evidence of psychological issues due to COVID in in order to analyse the prevalence of Anxiety, Depression and Insomnia among the affected population. The data analysis will help in crafting precautionary measures to mitigate the toxic mental health effects of COVID-19. 


\section{Material and Methods}

\section{Study design}

The study design was Systematic Review of published researched articles, extraction of data based on inclusion and exclu- sion criterion set and result interpretation after statistical analysis. The methodical literature review and selection criteria to extractCOVID-19 data was in accordance with the PRISMA statement depicted in Figure 1.

\section{PRISMA 2020 Flow Diagram}

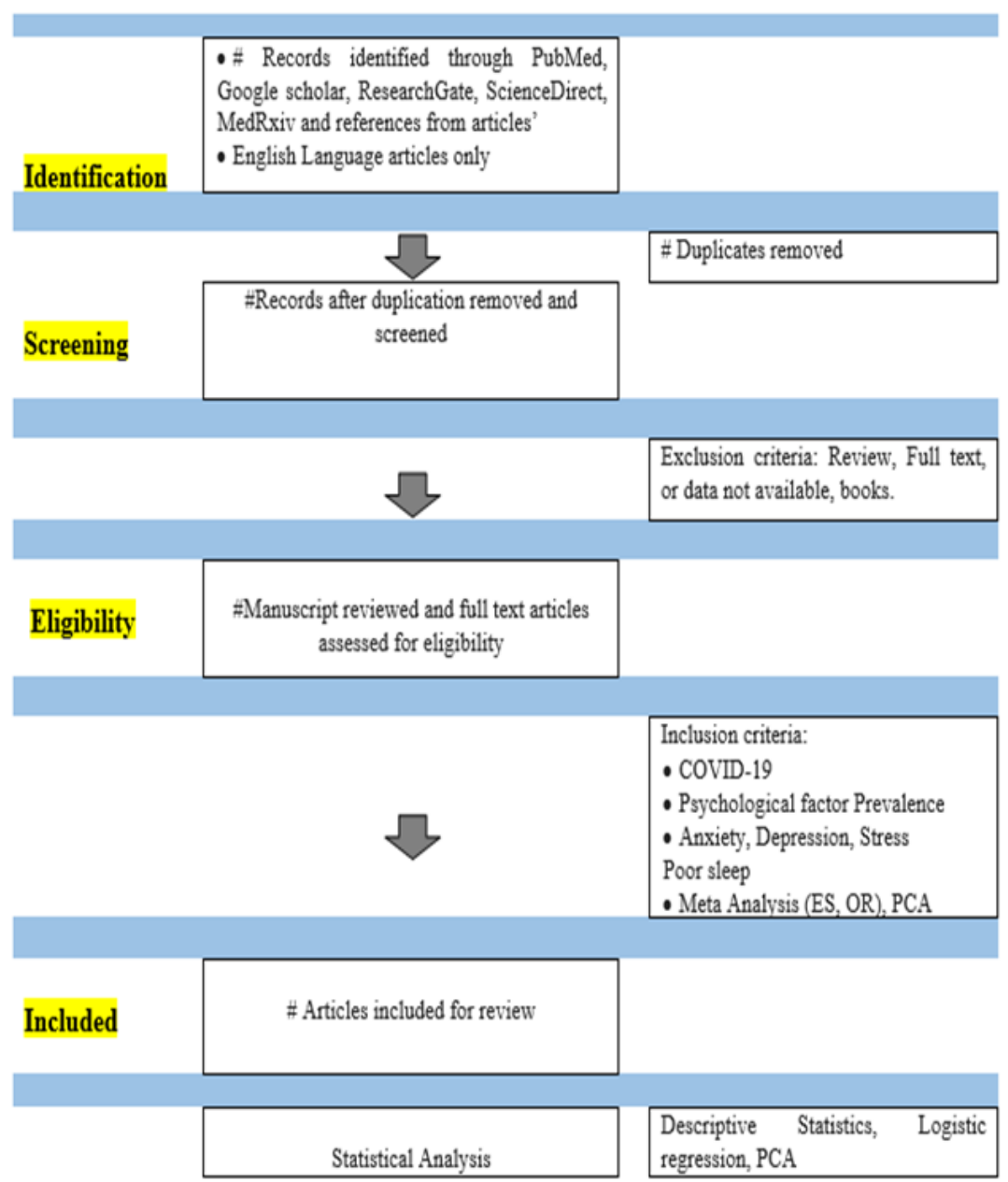

Figure 1: PRISMA Statement -Block diagram of the Study Design

\section{Data extraction}

The psychological data of anxiety, depression, poor sleep, suicidal cases linked to the COVID-19 positive patients, Quarantine population, medical professionals and general population were extracted from published articles on PubMed, Google scholar, Research gate, ScienceDirect, MedRxiv sites [5-13]. Further the references from articles were used to increase data base. Only English Language articles were searched and retrieved. The few of the total reviewed journals are listed in Table 1.

\section{Data synthesis and analysis}

Microsoft Excel for Windows was utilized in the current study to synthesize and organise data. The data was arranged as Author, publication and year, sample population, evaluation test methods applied, sample size and sample characteristics, age and gender, statistical method applied for assessment, psychological parameters determined and result outcomes. Details are presented in Table 1 . The primary psychological parameters retrieved were Anxiety, Depression, Insomnia, Distress (Figure 1). 


\section{Psychology and Behavioral Science International Journal}

Table 1: Review of literature on psychological distress related to Co-vid-19 pandemic.

\begin{tabular}{|c|c|c|c|c|c|c|c|c|}
\hline Year & $\begin{array}{l}\text { Publi- } \\
\text { cation }\end{array}$ & Author & $\begin{array}{c}\text { Study De- } \\
\text { sign }\end{array}$ & Subjects & Parameters & Evaluation Method & Statistics & Outcome \\
\hline $\begin{array}{c}24 \\
\text { Mar } \\
2020\end{array}$ & Online & $\begin{array}{c}\text { Xiangyu } \\
\text { Kong et al. } \\
\text { [5] }\end{array}$ & $\begin{array}{c}\text { Question- } \\
\text { naire Survey }\end{array}$ & $\begin{array}{l}\mathrm{N}=144 \\
\text { Hospi- } \\
\text { talized } \\
\text { COVID-19 } \\
\text { patients }\end{array}$ & $\begin{array}{c}\text { Depres- } \\
\text { sion and } \\
\text { anxiety } \\
\text { symp- } \\
\text { toms } \\
\text { - Social } \\
\text { support }\end{array}$ & $\begin{array}{c}\text { Hospital Anxiety } \\
\text { and Depression } \\
\text { Scale (HADS) } \\
\text { Perceived Social } \\
\text { Support Scale } \\
\text { (PSSS) }\end{array}$ & $\begin{array}{c}\text { Multivari- } \\
\text { ate linear } \\
\text { regression } \\
\text { analyses } \\
\\
\text { - } \quad \text { Bivariate } \\
\text { correlation }\end{array}$ & $\begin{array}{c}\text { 34.7\% Anx- } \\
\text { iety, } \\
\text { 28.4\% Depression } \\
\text { Less social } \\
\text { support was } \\
\text { correlated } \\
\text { with more } \\
\text { anxious } \\
\text { (r=-0.196, } \\
\mathrm{p}<0.05) \\
\text { and depres- } \\
\text { sive(r=-0.360 } \\
\text { ? } \mathrm{p}<0.05) \\
\text { symptoms }\end{array}$ \\
\hline $\begin{array}{c}31 \\
\text { Mar } \\
2020\end{array}$ & $\begin{array}{l}\text { General } \\
\text { Hospi- } \\
\text { tal } \\
\text { Psychi- } \\
\text { atry }\end{array}$ & $\begin{array}{l}\text { Jiang Du } \\
\text { et al. [6] }\end{array}$ & $\begin{array}{c}\text { Smart- } \\
\text { phone-based } \\
\text { survey }\end{array}$ & $\begin{array}{l}\mathrm{N}=134 \\
\text { Medical } \\
\text { Staff }\end{array}$ & $\begin{array}{c} \\
\text { Psycho- } \\
\text { logical } \\
\text { distress, } \\
\text { Psycho- } \\
\text { logical } \\
\text { prepared- } \\
\text { ness } \\
\text { Sleep } \\
\text { quality, } \\
\text { Stress, } \\
\text { Depres- } \\
\text { sion, } \\
\text { Anxiety }\end{array}$ & $\begin{array}{c}\text { Perceived Stress } \\
\text { Scale (PSS), } \\
\text { B } \quad \text { Beck Depression } \\
\text { Inventory-II (BDI-II), } \\
\text { B } \quad \text { Beck Anxiety } \\
\text { Inventory (BAI). }\end{array}$ & Logistic regression & $\begin{array}{l}\text { Prevalence of moderate } \\
\text { stress and elevated de- } \\
\text { pressive (BDI-II scores } \\
\geq 14 \text { ) and anxiety symp- } \\
\text { toms (BAI scores } \geq 8 \text { ): }\end{array}$ \\
\hline $\begin{array}{c}26 \\
\text { Mar } \\
2020\end{array}$ & $\begin{array}{l}\text { Psychi- } \\
\text { atry Re- } \\
\text { search }\end{array}$ & $\begin{array}{c}\text { Yeen } \\
\text { Huang et } \\
\text { al. [7] }\end{array}$ & $\begin{array}{l}\text { Web-based } \\
\text { cross-sec- } \\
\text { tional }\end{array}$ & $\begin{array}{l}\mathrm{N}=7,236 \\
\text { Medical } \\
\text { Staff, } \\
\text { Teach- } \\
\text { ers and } \\
\text { Others }\end{array}$ & $\begin{array}{l}\text { Gener- } \\
\text { alized } \\
\text { anxiety } \\
\text { disorder } \\
\text { (GAD), } \\
\text { - } \quad \text { Depres- } \\
\text { sive } \\
\text { symp- } \\
\text { toms } \\
\text { Sleep } \\
\text { quality. }\end{array}$ & $\begin{array}{c}\text { National Inter- } \\
\text { net Survey on } \\
\text { Emotional and } \\
\text { Mental Health } \\
\text { (NISEMH) } \\
\text { - } \quad \text { Generalized } \\
\text { Anxiety Disor- } \\
\text { der-7(GAD-7) } \\
\text { Center for Epi- } \\
\text { demiology Scale } \\
\text { for Depression } \\
\text { (CES-D) } \\
\text { PSQI (Pitts- } \\
\text { burgh Sleep } \\
\text { Quality Index) }\end{array}$ & $\begin{array}{c}\text { Chi-square } \\
\text { test } \\
\text { - Uni and } \\
\text { multi logistic } \\
\text { regression }\end{array}$ & $\begin{array}{l}\text { High prev- } \\
\text { alence of } \\
\text { GAD and } \\
\text { poor sleep } \\
\text { quality during } \\
\text { COVID-19 } \\
\text { outbreak }\end{array}$ \\
\hline
\end{tabular}




\section{Psychology and Behavioral Science International Journal}

\begin{tabular}{|c|c|c|c|c|c|c|c|c|}
\hline $\begin{array}{c}06 \\
\text { May } \\
2020\end{array}$ & $\begin{array}{l}\text { Brain, } \\
\text { Behav- } \\
\text { ior, and } \\
\text { Immu- } \\
\text { nity }\end{array}$ & $\begin{array}{c}\text { Sofia } \\
\text { Pappa et } \\
\text { al. [8] }\end{array}$ & Review & $\begin{array}{c}\mathrm{N}=33062 \\
\text { Medical } \\
\text { Staff }\end{array}$ & $\begin{array}{lc}\text { - } & \text { Anxiety } \\
\text { - } & \begin{array}{c}\text { Depres- } \\
\text { sion }\end{array} \\
\text { - } & \text { Insomnia } \\
\text { - } & \text { Pooled } \\
\text { Preva- } \\
\text { lence }\end{array}$ & $\begin{array}{c}\text { Z } \quad \begin{array}{c}\text { Zung Self-Rating } \\
\text { Anxiety Scale } \\
\text { (SAS) }\end{array} \\
\text { - } \quad \text { GAD-7 } \\
\text { Zung Self-Rat- } \\
\text { ing Depression } \\
\text { Scale (SDS) } \\
\text { Patient Health } \\
\text { Questionnaire } \\
\text { 9-item depres- } \\
\text { sion module } \\
\text { (PHQ-9) }\end{array}$ & $\begin{array}{l}\text { - } \quad \text { Metaanalysis } \\
\text { Random } \\
\text { effects model } \\
\text { Der Simoni- } \\
\text { an\& Laird) }\end{array}$ & $\begin{array}{c}\text { - High preva- } \\
\text { lence rates of } \\
\text { depression, } \\
\text { anxiety and } \\
\text { insomnia }\end{array}$ \\
\hline $\begin{array}{c}23 \\
\text { Mar } \\
2020\end{array}$ & $\begin{array}{l}\text { JAMA } \\
\text { Net- } \\
\text { work } \\
\text { Open }\end{array}$ & $\begin{array}{l}\text { Jianbo Lai } \\
\text { et al. [9] }\end{array}$ & $\begin{array}{l}\text { cross-sec- } \\
\text { tional hos- } \\
\text { pital-based } \\
\text { survey }\end{array}$ & $\begin{array}{c}\mathrm{N}=1257 \\
\text { Medical } \\
\text { Staff }\end{array}$ & $\begin{array}{c}\text { Depres- } \\
\text { sion, } \\
\text { Anxiety, } \\
\text { Insom- } \\
\text { nia, And } \\
\text { Distress } \\
\\
\text { - } \text { [factors } \\
\text { associat- } \\
\text { ed with } \\
\text { mental } \\
\text { health } \\
\text { out- } \\
\text { comes] }\end{array}$ & $\begin{array}{lc}\text { - } & \text { PHQ-9, GAD-7 } \\
\text { - } \quad \text { 7-item Insomnia } \\
\text { Severity Index } \\
\text { (ISI) } \\
\text { - } \\
\text { 22-item Impact } \\
\text { of Event Scale- } \\
\text { Revised (IES-R) }\end{array}$ & $\begin{array}{l}\text { - } \quad \text { Multivari- } \\
\text { able logistic } \\
\text { regression }\end{array}$ & $\begin{array}{c}\text { - } \quad \text { Participants } \\
\text { reported } \\
\text { experiencing } \\
\text { psychological } \\
\text { burden } \\
\text { - Frontline } \\
\text { medical staff } \\
\text { at higher risk } \\
\text { of mental } \\
\text { health symp- } \\
\text { toms }\end{array}$ \\
\hline $\begin{array}{c}14 \\
\text { Apr } \\
2020\end{array}$ & $\begin{array}{l}\text { Fron- } \\
\text { tiers in } \\
\text { Psychi- } \\
\text { atry }\end{array}$ & $\begin{array}{l}\text { Chenxi } \\
\text { Zhang et } \\
\text { al. [10] }\end{array}$ & $\begin{array}{l}\text { WeChat } \\
\text { program } \\
\text { Question- } \\
\text { naire }\end{array}$ & $\begin{array}{c}\mathrm{N}=1,563 \\
\text { Medical } \\
\text { Staff }+ \\
\text { others }\end{array}$ & $\begin{array}{l}\text { - Insomnia, } \\
\text { depres- } \\
\text { sive, anx- } \\
\text { iety and } \\
\text { stress-re- } \\
\text { lated } \\
\text { symp- } \\
\text { toms }\end{array}$ & $\begin{array}{ll}\text { - } & \text { PHQ-9, GAD-7, } \\
\text { ISI-7, IES-R-22 }\end{array}$ & $\begin{array}{c}\text { - } \quad \text { Multiple Bi- } \\
\text { nary Logistic } \\
\text { Regression }\end{array}$ & $\begin{array}{l}\text { Insomnia } \\
\text { symptoms } \\
\text { during the } \\
\text { COVID-19 } \\
\text { outbreak }\end{array}$ \\
\hline $\begin{array}{c}09 \\
\text { Apr } \\
2020\end{array}$ & $\begin{array}{l}\text { Psy- } \\
\text { choth- } \\
\text { erPsy- } \\
\text { chosom }\end{array}$ & $\begin{array}{c}\text { Wen-rui } \\
\text { Zhang et } \\
\text { al. [11] }\end{array}$ & $\begin{array}{l}\text { Online } \\
\text { survey }\end{array}$ & $\mathrm{N}=2,182$ & $\begin{array}{l}\text { - } \quad \text { insomnia, } \\
\text { anxiety, } \\
\text { depres- } \\
\text { sion, } \\
\text { somatiza- } \\
\text { tion } \\
\\
\text { obses- } \\
\text { sive-com- } \\
\text { pulsive } \\
\text { symp- } \\
\text { toms, } \\
\text { phobic } \\
\text { anxiety }\end{array}$ & $\begin{array}{cc}\text { • } & \text { ISI-7 } \\
\text { • } & \text { PHQ-4 } \\
\text { - } & \text { GAD-2 } \\
\text { - } & \text { Symptom Check } \\
\text { List-90- } \\
\text { - } \\
\text { revised (SCL- } \\
90-\mathrm{R})\end{array}$ & $\begin{array}{l}\text { Chi square } \\
\text { and } \\
\text { Mann-Whit- } \\
\text { ney tests }\end{array}$ & $\begin{array}{c}\text { During } \\
\text { COVID-19 } \\
\text { medical } \\
\text { health work- } \\
\text { ers had } \\
\\
\text { psychosocial } \\
\text { problems and } \\
\text { risk factors } \\
\text { for develop- } \\
\text { ing them. }\end{array}$ \\
\hline $\begin{array}{c}16 \\
\text { Mar } \\
2020\end{array}$ & Online & $\begin{array}{l}\text { Zhou Zhu } \\
\text { et al. [12] }\end{array}$ & $\begin{array}{l}\text { Online ques- } \\
\text { tionnaire } \\
\text { survey }\end{array}$ & $\mathrm{N}=5062$ & $\begin{array}{c}\text { Depres- } \\
\text { sive, anx- } \\
\text { iety and } \\
\text { stress-re- } \\
\text { lated } \\
\text { symp- } \\
\text { toms }\end{array}$ & $\begin{array}{l}\text { PHQ-9, GAD-7, } \\
\text { ISI-7, IESR-22 }\end{array}$ & $\begin{array}{l}\text { Multivari- } \\
\text { ate logistic } \\
\text { regression }\end{array}$ & $\begin{array}{c}\text { Medical } \\
\text { staff facing } \\
\text { a serious } \\
\text { psychological } \\
\text { challenge }\end{array}$ \\
\hline $\begin{array}{c}07 \\
\text { Apr } \\
2020\end{array}$ & $\begin{array}{l}\text { Annals } \\
\text { of In- } \\
\text { ternal } \\
\text { Medi- } \\
\text { cine }\end{array}$ & $\begin{array}{l}\text { Benjamin } \\
\text { YQ Tan et } \\
\text { al. [13] }\end{array}$ & $\begin{array}{l}\text { question- } \\
\text { naire survey }\end{array}$ & $\begin{array}{c}\mathrm{N}=470 \\
\text { Medical } \\
\text { Staff }+ \\
\text { others }\end{array}$ & $\begin{array}{c}\text { Psycho- } \\
\text { logical } \\
\text { distress, } \\
\text { depres- } \\
\text { sion, } \\
\text { - anxiety, } \\
\text { and stress }\end{array}$ & $\begin{array}{c}\text { Depression, } \\
\text { Anxiety, and } \\
\text { Stress Scales } \\
\text { (DASS-21) } \\
\text { - } \quad \text { Impact of Events } \\
\text { Scale-Revised } \\
\text { (IES-R) } \\
\text { - } \quad \text { PTSD }\end{array}$ & $\begin{array}{c}\text { Pearson } \\
\text { chi }^{2} \text { test and } \\
\text { Student } t \\
\text { test } \\
\text { - Multivariable } \\
\text { regression }\end{array}$ & $\begin{array}{l}\text { Risk for psy- } \\
\text { chological dis- } \\
\text { tress during } \\
\text { the COVID-19 } \\
\text { outbreak. }\end{array}$ \\
\hline
\end{tabular}




\section{Psychology and Behavioral Science International Journal}

\section{Results and Discussion}

The statistics of the included sample in the study is shown in the descriptive statistics in Table 2. A total of $n=18,048$ samples from the 8 studies where included in the analysis. All the studies reported the psychological factors like anxiety, depression, stress and insomnia in medical staff and general population during the COVID-19 pandemic. Some studies that were reviewed had also reported cases of PSTD, Suicide, fear and social connect factor related to lockdown. Out of 8 studies 6 was in mainland china and one was in Wuhan city and one was in Singapore (Figure 2).

Table 2: Descriptive statistics of sample population.

\begin{tabular}{|c|c|c|c|c|c|c|c|c|}
\hline Item & Sample n & Age & Male & Female & Anxiety & Depression & Stress/ Distress & Insomnia / Poor Sleep \\
\hline Mean & 2256 & 36.6 & 807.8 & 1273.1 & 670.8 & 483.9 & 449.9 & 381.339 \\
\hline SD & 2572.1 & 6.8 & 1070.3 & 1791.1 & 861.4 & 505.6 & 630.4 & 480.728 \\
\hline Skewness & 1.339 & 0.95 & 2.16 & 1.333 & 1.744 & 0.955 & 0.902 & 1.116 \\
\hline SE of Skewness & 0.752 & 0.75 & 0.75 & 0.752 & 0.752 & 0.752 & 0.752 & 0.752 \\
\hline Minimum & 134 & 28 & 53 & 1.401 & 4.96 & 5.76 & 0.001 & 0.001 \\
\hline Maximum & 7236 & 49.98 & 3284 & 4304 & 2540 & 1454 & 1509 & 1317 \\
\hline
\end{tabular}

\section{$\%$ psychological factors influenced due to COVID-19}

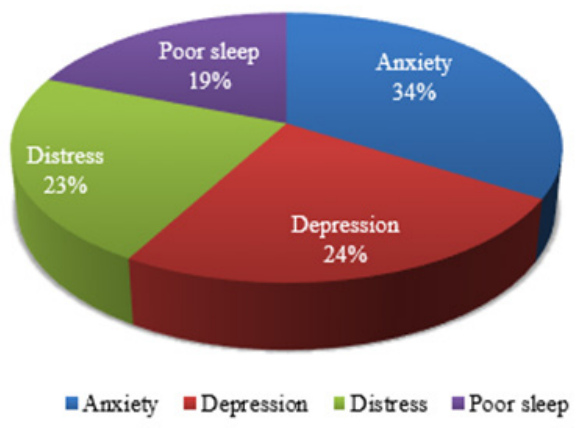

Figure 2: Pie Chart showing \% of Anxiety, Depression, Distress and Poor sleep impacting sample population due to COVID-19.

The multiple linear regression analysis (Table 3) showed that sample type $(\beta=-0.41, p<0.05)$, Sample number $(\beta 0.61, p<0.01)$, age $(\beta=-0.30, p<0.05)$, and gender $/$ male $(\beta=0.58, p<0.01)$ were

associated with anxiety for COVID-19. As compared to anxiety, depression and stress affected people due to COVID-19 to a lesser degree.

Table 3: Multivariate regression analysis of factors associated with anxiety, depression and distress.

\begin{tabular}{|c|c|c|c|c|c|c|}
\hline Anxiety & Beta & Std.Err. & B & Std.Err. & $t(2)$ & p-level \\
\hline Sample Type & -0.41 & 0.05 & -487.91 & 63.29 & -7.71 & 0.02 \\
\hline Sample $\mathrm{n}$ & 0.61 & 0.06 & 0.20 & 0.02 & 10.30 & 0.01 \\
\hline Age & -0.30 & 0.04 & -39.15 & 5.68 & -6.89 & 0.02 \\
\hline Male & 0.58 & 0.07 & 0.46 & 0.05 & 8.67 & 0.01 \\
\hline \multicolumn{7}{|c|}{ Anxiety $\mathrm{R}=.99919257 \mathrm{R}^{2}=.99838579$ Adjusted $\mathrm{R}^{2}=.99515737 \mathrm{~F}(4,2)=309.25 \mathrm{p}$} \\
\hline Depression & Beta & Std.Err. & B & Std.Err. & $t(2)$ & p-level \\
\hline Sample Type & -0.42 & 0.31 & -286.18 & 209.12 & -1.37 & 0.30 \\
\hline Sample $\mathrm{n}$ & 0.47 & 0.34 & 0.09 & 0.07 & 1.38 & 0.30 \\
\hline Age & -0.56 & 0.25 & -41.31 & 18.77 & -2.20 & 0.16 \\
\hline Male & 0.59 & 0.38 & 0.27 & 0.17 & 1.53 & 0.27 \\
\hline
\end{tabular}




\section{Psychology and Behavioral Science International Journal}

\begin{tabular}{|c|c|c|c|c|c|c|}
\hline Distress & Beta & Std.Err. & B & Std.Err. & t(2) & p-level \\
\hline Sample Type & -0.38 & 0.74 & -326.48 & 645.68 & -0.51 & 0.66 \\
\hline Sample $\mathrm{n}$ & 1.43 & 0.82 & 0.35 & 0.20 & 1.74 & 0.22 \\
\hline Age & -0.87 & 0.61 & -82.05 & 57.97 & -1.42 & 0.29 \\
\hline Male & -1.39 & 0.92 & -0.81 & 0.54 & -1.51 & 0.27 \\
\hline \multicolumn{7}{|c}{} \\
\hline
\end{tabular}

As can be seen from the present analysis that Anxiety, Depression, Stress and Insomnia, amongst other are critical psychological parameters that are affected during painful physical or mental conditions of COVID-19 Pandemic. These four primary parameters of current study that were critically affected during pandemic, were subjected to principal component analysis (PCA). The analysis method of PCA is a dimension reduction procedure by combining a large number of parameters into a smaller set of components based on their correlation or covariance. The PCA analysis was carried out using JASPv0.12.2 and its Scree plot is depicted in Figure 3. The Scree plot shows "elbow" at 2 components. Two principal components PC1 with eigenvalue $(\varepsilon=2.67)$ and PC2 having eigenvalue $(\varepsilon=1.11)$ were derived. The Path -Analysis plot of is presented in Figure 4. The loadings on the first component PC1 are all positive and loaded across three variables Anxiety, Depression and Insomnia. PC2 have positive and negative loading.

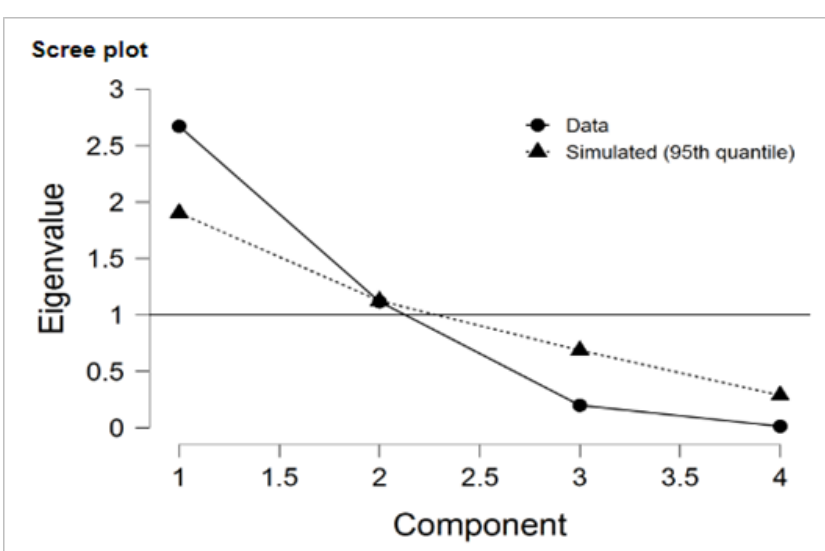

Figure 3: Scree plot of psychological factor analysis of the Anxiety, Depression, Distress and Insomnia.

\section{Path Diagram}

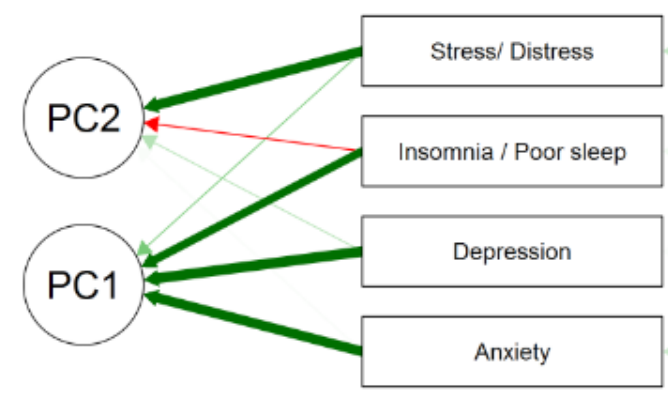

Figure 4: Principal Component Path Analysis identified a two-component structure PC1 and PC2

\section{Conclusion}

This review indicates that there are many psychological aspects that are affected during the prevailing pandemic which have been evaluated in recent studies. Data analysis reveals that there is considerable evidence to conclude that patients of COVID-19 and general population experienced anxiety and felt unsafe during corona virus pandemic peak period. The Principal Component Analysis allowed, critical Psychological parameters 
Anxiety, Depression, Stress and Insomnia to be condensed to two factors PC1 and PC2, for assessing COVID-19 mental health. Besides mental health parameters shooting up, there were confirmed cases of suicide under distress of Corona virus which presents an alarming situation. The present study recommends paying added attention towards the mental health of patients, frontline health care workers, in terms of providing good quality protection gears, psychological support and psychological prescription module as well as strong family supportduring and post theCOVID-19 epidemic.It also warrants appropriate professional counselling wherever necessary.

\section{References}

1. Coronavirus. (2020) World Health Organization.

2. Sritharan, Jeavana, Sritharan Ashvinie (2020) Emerging Mental Health Issues from the Novel Coronavirus (COVID-19) Pandemic. In: Journal of Health and Medical Sciences 3(2): 157-162.

3. Lai J, Ma S, Wang Y, Wang Y, Cai Z, et al. (2020) Factors associated with mental health outcomes among health care workers exposed to coronavirus disease 2019. JAMA Network Open 3(3): e203976.

4. Fardin MA (2020) COVID-19 and Anxiety: A Review of Psychological Impacts of Infectious Disease Outbreaks. Arch Clin Infect Dis.

5. Kong X, Zheng K, Tang M, Kong F, Zhou J, et al. (2020) Prevalence and Factors Associated with Depression and Anxiety of Hospitalized Patients with COVID-19.

6. Du J, Dong L, Wang T, Chenxin Yuan, Rao Fu, et al. (2020) Psychological symptoms among frontline healthcare workers during COVID-19 outbreak in Wuhan. Gen Hosp Psychiatry.

7. Huang Y, Zhao N (2020) Generalized anxiety disorder, depressive symptoms and sleep quality during COVID-19 outbreak in China: a web-based cross-sectional Survey. PII: S0165-1781(20): 30607-7.
8. Pappaet S, Ntellac V, Gianna T, Giannakoulisc VG, Papouts E, et al. (2020) Prevalence of depression, anxiety, and insomnia among healthcare workers during the COVID-19 pandemic: A systematic review and meta-analysis. Brain, Behavior, and Immunity.

9. Laiet J, Simeng Ma, Wang Y, Cai Z, Hu J (2020) Factors Associated With Mental Health Outcomes Among Health CareWorkersExposed to Coronavirus Disease 2019. JAMA Network Open. 2020;3(3): e203976.

10. Zhang C (2020) Survey of Insomnia and Related Social Psychological Factors Among Medical Staff Involved in the 2019 Novel Coronavirus Disease Outbreak. Frontiers in Psychiatry. 11: 306.

11. Wen-rui Zhang, Wang K, Yin L, Zhao W, et al. (2020) Mental Health and Psychosocial Problems of Medical Health Workers during the COVID-19 Epidemic in china. Psychother Psychosom.

12. Zhu Z, Xu S, Wang H, Liu Z, Wu J, et al. (2020) COVID-19 in Wuhan: Immediate Psychological Impact on 5062 Health Workers. Med Rxiv preprint.

13. Benjamin YQ Tan, Nicholas WS, Chew, Grace KH Lee, MD et al. (2020) Psychological Impact of the COVID-19 Pandemic on Health Care Workers in Singapore. Annals of Internal Medicine.

14. Shacham M, Raz YM, Kolerman R, Mijiritsky O, Ben-Ezra M, et al. (2020) COVID-19 Factors and Psychological Factors Associated with Elevated Psychological Distress among Dentists and Dental Hygienists in Israel. Int. J. Environ. Res. Public Health 17: 2900.

15. Wei-jie Guan, Wen-hua Liang, Yi Zhao, Heng-rui Liang, Zi-sheng Chen, et al. (2020) Comorbidity and its impact on 1590 patients with COVID-19 in China: a nationwide analysis. EurRespir J 55: 2000547.

16. Zhang Y, Ma ZF (2020) Impact of the COVID-19 Pandemic on Mental Health and Quality of Life among Local Residents in Liaoning Province, China: A Cross-Sectional Study. Int J Environ Res. Public Health 17: 2381.

17. Griffiths MD, Mamun MA (2020) COVID-19 suicidal behavior among couples and suicide pacts: Case study evidence from press reports. Psychiatry Research 289:113105.

\section{Your next submission with Juniper Publishers will reach you the below assets}

- Quality Editorial service

- Swift Peer Review

- Reprints availability

- E-prints Service

- Manuscript Podcast for convenient understanding

- Global attainment for your research

- Manuscript accessibility in different formats

( Pdf, E-pub, Full Text, Audio)

- Unceasing customer service

Track the below URL for one-step submission https://juniperpublishers.com/online-submission.php 